\title{
Helminth infection-induced malignancy
}

\author{
Paul J. Brindley ${ }^{1,2 *}$, Alex Loukas ${ }^{3 *}$ \\ 1 Department of Microbiology, Immunology and Tropical Medicine, School of Medicine \& Health Sciences, \\ The George Washington University, Washington DC, United States of America, 2 Research Center for \\ Neglected Tropical Diseases of Poverty, School of Medicine \& Health Sciences, The George Washington \\ University, Washington DC, United States of America, 3 Centre for Biodiscovery and Molecular Development \\ of Therapeutics, Australian Institute of Tropical Health \& Medicine, James Cook University, Cairns, \\ Queensland, Australia \\ * pbrindley@gwu.edu (PJB); alex.loukas@jcu.edu.au (AL)
}

\section{Infection with some helminth pathogens represents a biological carcinogen}

Infectious diseases cause more than $20 \%$ of cancers in the developing world [1]. About a dozen pathogens including Epstein-Barr virus and human T cell lymphocytotropic virus 1 are among the well-known examples. In addition, infection with several trematodes, which are eukaryotes, can cause malignancy. The International Agency for Research on Cancer categorizes infection with the fish-borne trematodes Opisthorchis viverrini and Clonorchis sinensis and the blood fluke Schistosoma haematobium as Group 1 biological carcinogens [2]. In addition to parasitism directly damaging development, health, and prosperity of infected populations, infection with these helminths leads to cholangiocarcinoma (CCA) (bile duct cancer) and squamous cell carcinoma (SCC) of the urinary bladder, respectively [2]. By contrast, infection with phylogenetic relatives, also trematodes of the phylum Platyhelminthes and also major pathogens, is not carcinogenic. These irregularities suggest that either helminth-specific metabolites contribute to tumorigenesis and/or that certain tissues or organs are particularly susceptible to infection-induced malignancy. Moreover, each of these helminth infections must be viewed holistically in the context of a perfect storm of risk for cancer (see [3]).

Copyright: ๑ 2017 Brindley, Loukas. This is an open access article distributed under the terms of the Creative Commons Attribution License, which permits unrestricted use, distribution, and reproduction in any medium, provided the original author and source are credited.

Funding: AL acknowledges support from NHMRC via a Senior Principal Research Fellowship, project and program grants. Support from the National Institute of Allergy and Infectious Diseases (NIAID), Tropical Medicine Research Center award number P50Al098639, and the National Cancer Institute (NCl) award number R01CA164719 is gratefully acknowledged. The funders had no role in study design, data collection and analysis, decision to publish, or preparation of the manuscript.

Competing interests: The authors have declared that no competing interests exist.

\section{Helminth infection-induced cancers \\ $O$. viverrini and $C$. sinensis infection-induced cholangiocarcinoma}

Infection is accomplished by ingestion of undercooked freshwater fish infected with the metacercaria stage of these species of liver flukes. Human infection leads to hepatobiliary disease, including cholangitis and periductal fibrosis. In liver fluke-endemic regions, infection is the major risk factor for CCA $[2,4,5]$. CCAs are slow-growing adenocarcinomas that metastasize to distant sites due to proximity to lymphatic vessels. Liver fluke-related CCA is often diagnosed at an advanced stage, when the primary cancer is no longer amenable to curative surgery [5]. The mechanism(s) by which infection initiates genetic lesions that eventually culminate in CCA is not understood, although it is likely to be multifactorial, involving biliary tract and systemic chronic inflammation and associated endogenous nitrosation [6,7], secretion of mitogens and other mediators by the parasite, and cofactors including dietary preferences for nitrosamines-rich foods $[4,8,9]$ (Fig 1$)$. 


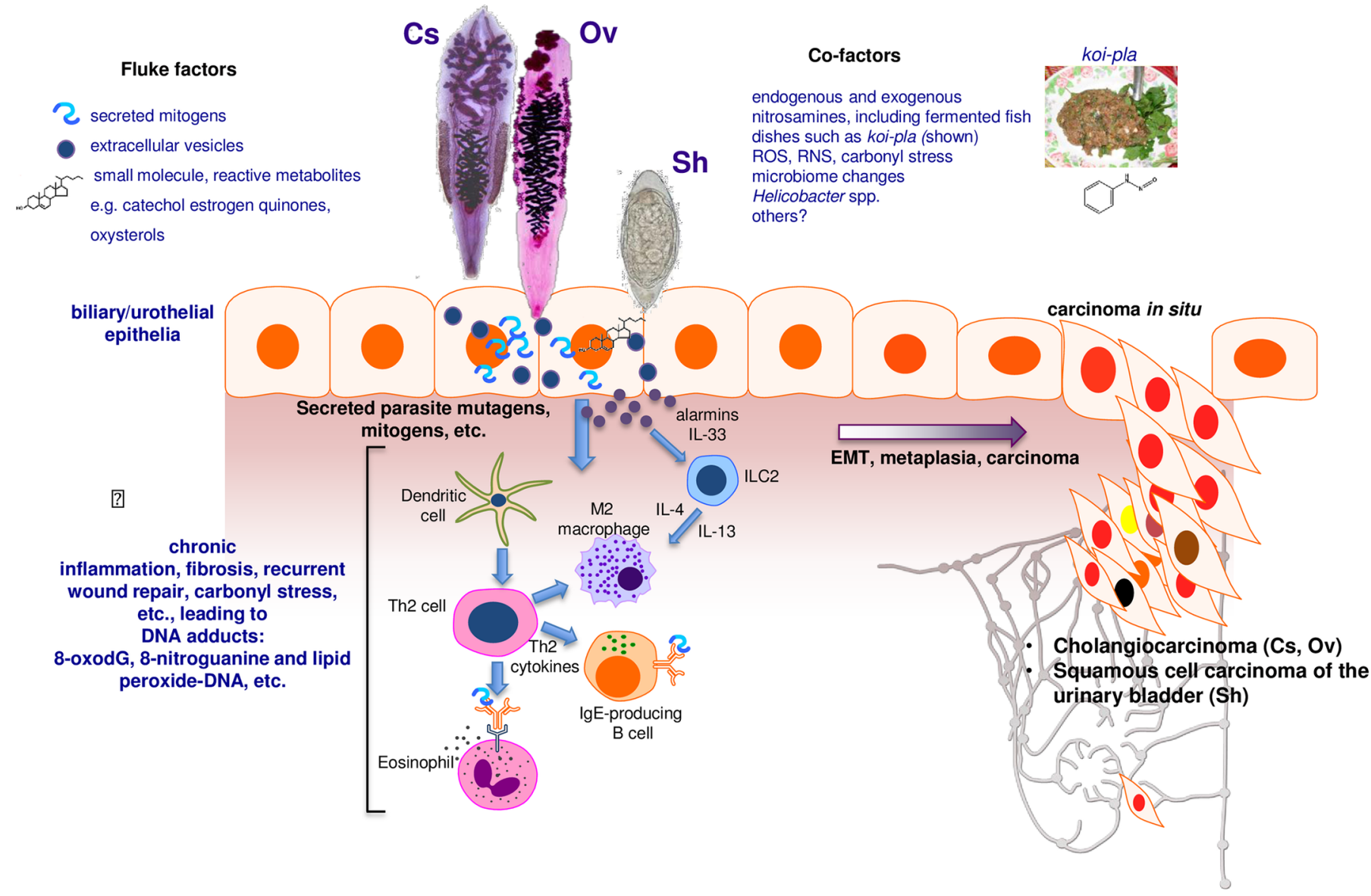

Fig 1. Schematic representation of hypothesized processes of carcinogenesis of the biliary tract and urinary bladder during chronic infection with fish-borne liver flukes Opisthorchis viverrini (Ov) and Clonorchis sinensis (Cs) and the blood fluke Schistosoma haematobium (Sh). Photomicrographs: adult developmental stages of $\mathrm{Cs}$ and Ov and the egg stage of Sh. During infection, mutations initiate carcinogenesis, perhaps as the consequence of interaction of epithelial cell chromosomal DNA with inflammation-associated reactive oxygen species (ROS) and reactive nitrogen species (RNS) and lipid peroxidation and/or metabolites released by the worms, such as catechol estrogen quinones and oxysterols. Subsequently, mediators of helminth origin such as granulin from Ov promote epidermal to mesenchymal transition (EMT), transformed cell growth, complementary angiogenesis, down-regulation of apoptosis, and other hallmarks of cancer.

\section{Urogenital schistosomiasis and bladder cancer}

Three major species of schistosomes are the agents of schistosomiasis: S. japonicum and $S$. mansoni cause intestinal schistosomiasis whereas S. haematobium causes urogenital schistosomiasis. Of about 112 million cases of S. haematobium infection in sub-Saharan Africa, 70 million are associated with hematuria, 18 million with major bladder wall pathology, and 10 million with hydronephrosis leading to kidney damage. Deposition of ova of S. haematobium in the bladder wall can lead to SCC of the bladder $[2,10]$.

\section{Initiation, promotion, and progression of tumorigenesis}

Where opisthorchiasis is endemic, people can remain infected for decades. Opisthorchiasis provokes inflammation of the biliary tree, with hyperplasia and metaplasia of the cholangiocytes that line the biliary tract adjacent to the flukes. Opisthorchiasis-induced fibrosis engulfs the proliferating cells, manifesting as biliary periductal fibrosis [4, 11]. Chronic inflammation in response to parasite metabolites and growth factors is implicated in the inflammatory 
response linked with infection [11]. Additional factors including carriage of Helicobacter and other microbiome changes within the biliary tract might participate $[8,12]$. Elevated plasma interleukin-6 (IL-6) is associated with marked increase in risk of periductal fibrosis during opisthorchiasis and compounds pathogenesis by promoting a fibrogenic inflammatory milieu. The cell of origin of CCA is the cholangiocyte, a specialized epithelial cell that lines the bile duct. Following initiation, for example by oxidation of cholangiocyte chromosomal DNA by oxysterols generated by reactive oxygen species (ROS) and reactive nitrogen species (RNS) arising during opisthorchiasis-induced oxidative stress [13] and/or oxysterols released by the liver fluke $[8,14]$, oncogenesis appears to be promoted by cholestasis and chronic inflammation. The release and downstream consequences of IL-6, platelet-derived growth factor, tumor necrosis factor-alpha (TNF- $\alpha$ ), and transforming growth factor-beta (TGF- $\beta$ ) are pivotal to the proliferation of cholangiocytes. Autonomous proliferation, evasion of apoptosis, and angiogenesis sustain the incipient neoplasm [11].

Bladder cancer is the most common tumor of the urinary system and consists of 2 main forms-urothelial carcinoma (UCC) and SCC. The bladder is lined by a specialized epithelium termed the urothelium, which is exposed routinely to potential carcinogens and hence is at particular risk of cancer. The urothelium is a stratified epithelium composed of keratin 5 (K5)expressing basal cells, intermediate cells, and umbrella cells. K5-positive basal cells likely give rise to SCC [10, 15-17]. UCC accounts for around 90\% of bladder cancers, and major risk factors include tobacco smoking, occupational exposure to aromatic amines and polycyclic hydrocarbons, and bladder stones, among others $[10,15]$. In regions endemic for urogenital schistosomiasis, SCC is more common than UCC and may be the cancer of highest incidence $[2,10]$.

Schistosome eggs entrapped in the bladder wall release metabolites, presumably to facilitate egress of the egg to the lumen and to the external environment. The process leads to hematuria and to chronic inflammation, in turn increasing the risk of urothelial hyperplasia, dysplasia, and SCC (Fig 1). Urogenital schistosomiasis (UGS) is a chronic infection, often interrupted by drug treatment and often followed in turn by reinfection. Fibrosis induced by entrapped schistosome eggs may promote cellular proliferation, hyperplasia, and metaplasia that eventually induce carcinogenesis [10]. Mass spectrometric analysis of urine during UGS reveals estrogenlike metabolites including catechol estrogen quinones (CEQ), likely of schistosome origin, CEQ-DNA adducts, and novel metabolites derived from 8-oxo-7, 8-dihydro-2'-deoxyguanosine (8-oxodG) [18]. Nitrosamines and increased levels of beta-glucuronidase and cyclooxygenase- 2 derived from schistosomes also represent potential bladder carcinogens. These helminth infection-derived carcinogens may damage DNA, leading to somatic mutations in oncogenes such as $p 53$, retinoblastoma protein, epidermal growth factor receptor, and erbB2 receptor tyrosine kinase. In like fashion, chromosomal adducts including 8-oxodG and 8-nitroguanine and lipid peroxide-DNA increase during opisthorchiasis (see [19]).

Investigation of UGS-induced bladder cancer is challenging given that rodent models do not exhibit urogenital disease; infection with S. haematobium causes hepatointestinal disease in rodents. However, in a recently developed rodent model, eggs of $S$. haematobium injected into the bladder wall of mice provoke egg-associated pathogenesis more reflective of the human condition [20, 21]. Preneoplastic lesions involving epithelial to mesenchymal (EMT)like profiles have been described in this model following coadministration of exogenous nitrosamines [22]. These approaches might lead to deeper understanding of the carcinogenesis of UGS-induced SCC. A hamster model involving coadministration of nitroso compounds and liver fluke infection has long been employed to study infection-induced bile duct cancer [2, 4, $11,19,23]$. Curiously, among the human schistosomes, only infection with S. haematobium is categorized as a biological carcinogen [2]. Perhaps local levels of metabolites such as CEQ are 
not produced by S. mansoni and S. japonicum and/or the hepatointestinal niche of these intestinal disease-causing schistosomes is less disposed to schistosome infection-induced malignancy.

\section{Mutations, mutational signatures, rearrangements, epigenetics}

Cancer arises when mutations occur in the DNA of the genome of the target cell and the mutations lead to uncontrolled cellular proliferation, invasion, and metastasis. The landscape of mutations that accumulate within the tumor record the mutagenic processes that have taken place over the life span of the malignancy. Each mutation endows an imprint on the genome of the tumor, documenting the types of DNA lesion and repair processes that lead to base substitutions, insertions, deletions, and structural variations. These mutational profiles have implications for diagnosis, therapy, and public health interventions.

Metabolites of helminth origin, including oxysterols, catechol estrogens, heme from ingested blood, and others, all of which are reactive species, may depurinate host cell DNA, leading to error-prone repair that results in mutations of cancer driver genes [8, 18]. At the genomic level, analysis of the mutation profiles of $O$. viverrini-related versus nonliver flukeinduced CCA reveals marked variation in mutation patterns [24]. Somatic mutations occur frequently in the tumor suppressor genes $p 53$ and smad4 in O. viverrini-induced CCA. By contrast, somatic mutations in the genes encoding BRCA1 associated protein- 1 and isocitrate dehydrogenases 1 and 2 are more common in non-O. viverrini-associated CCA [24, 25]. Mutations in $p 53$ and smad4 directly affect the p53- and TGF-signaling pathways, both of which are involved in tumorigenesis. Thus, distinct causes of CCA induce discrete somatic alterations, even within the same cancer type [25]. Emphasizing this point, liver fluke infection-induced CCA exhibits altered DNA methylation and transcriptional profiles reflective of xenobiotic metabolism and pro-inflammatory responses in comparison to nonliver fluke infection-induced CAA and to healthy biliary duct [24-26].

Details also have emerged from whole exome sequences of UCC, although the specific mutational landscape of SCC and hence UGS-induced SCC have not been reported [2, 15]. Mutation of sonic hedgehog can initiate carcinogenesis in bladder cancer [16]. Recurrent mutations occur in $>30$ other genes involved in cell proliferation, differentiation, genetic stability, and specifically cell-cycle regulation, chromatin regulation, and kinase signaling pathways [27]. Bladder cancer frequently exhibits $\mathrm{C}$ to $\mathrm{T}$ or $\mathrm{C}$ to $\mathrm{G}$ mutations at $\mathrm{TC}$ dinucleotides, which may reflect hyperactive DNA editing by apolipoprotein B mRNA-editing enzyme, catalytic polypeptide-like (APOBEC) cytidine deaminases [28].

\section{Host-parasite interactions}

Communication between helminths and host cells likely evolved to facilitate parasitism. Communicating metabolites may, however, contribute to carcinogenesis $[8,29]$. For example, $O$. viverrini secretes a growth factor termed $O v$-GRN-1 that shares homology with human granulin [30]. Secreted $O v$-GRN-1 may promote angiogenesis and also wound repair of the bile ducts damaged by the liver fluke $[11,30]$ (Fig 2). Moreover, Ov-GRN-1 may mimic the action of interleukin-33 (IL-33), an epithelial mitogen for cholangiocytes, in the development of CCA [31]. IL-33 primes type 2 innate lymphoid cells to induce proliferation of neighboring cholangiocytes by the release of Interleukin-13 (IL-13). Antibodies to $O v$-GRN-1 blocked its ability to drive proliferation of host cells [30,32]. Liver flukes also secrete extracellular vesicles (EVs) [33] (Fig 1). The membranes surrounding EVs are enriched in tetraspanins, proteins that interact with transmembrane and cytosolic signaling proteins [34]. Cholangiocytes internalize both $O$. viverrini EVs and $O v$-GRN-1 [32]. Antibodies to a tetraspanin located on the 

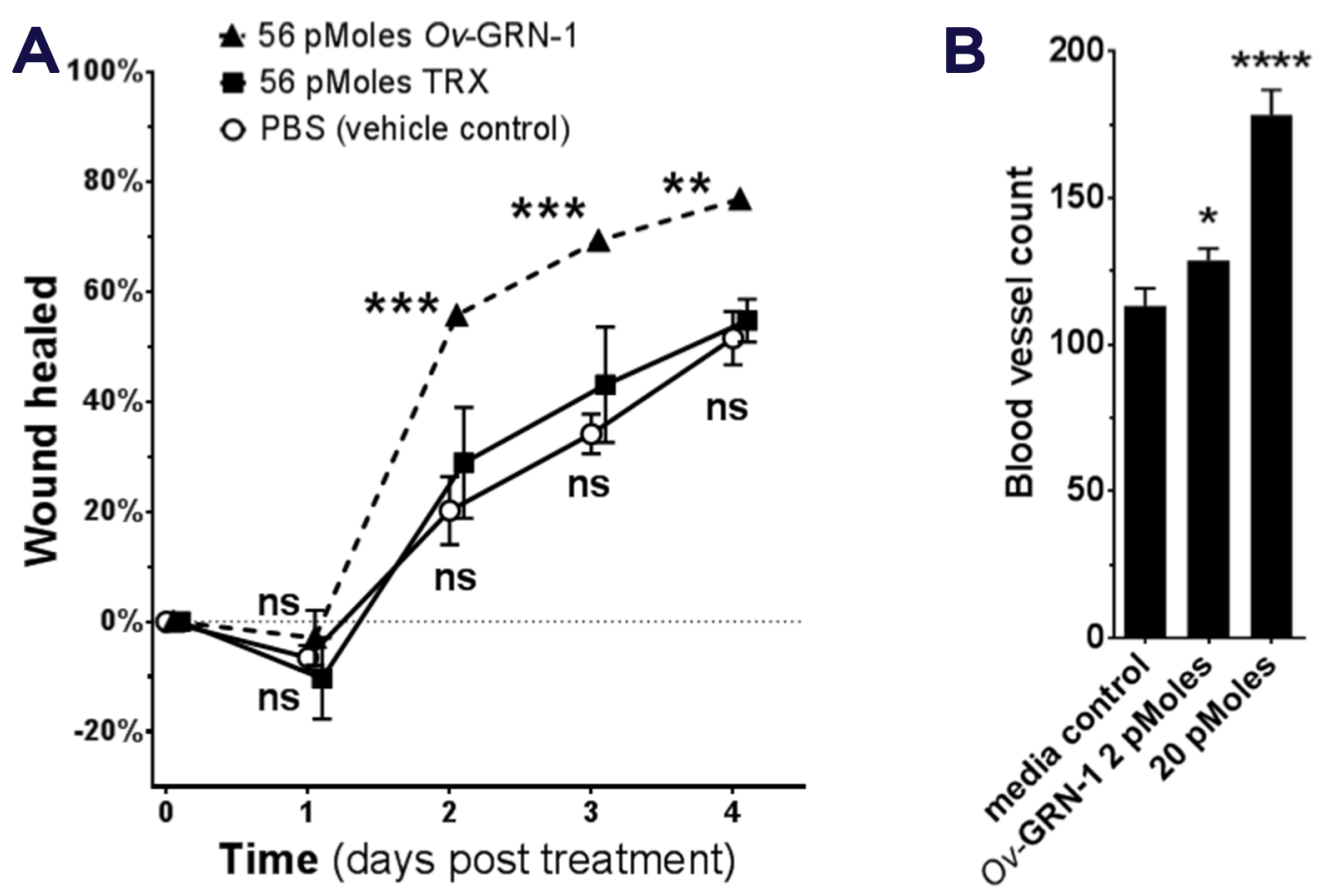

Fig 2. Liver fluke granulin promotes wound repair and angiogenesis. Opisthorchis viverrini secretes a growth factor termed liver fluke granulin, $O v$-GRN-1, an orthologue of mammalian granulin. Ov-GRN-1 stimulates cholangiocytes to proliferate and promotes wound healing and angiogenesis. A recent report on Ov-GRN-1 [32] illustrates some of these carcinogenic-conducive properties of the growth factor, as follows: (A) rate of wound closure on a cutaneous lesion on mice, (B) the angiogenic nature of $O v-G R N-1$ revealed using the chorioallantoic membrane assay in fertilized quail eggs.

https://doi.org/10.1371/journal.ppat.1006393.g002

surface of O. viverrini EVs block the internalization by cholangiocytes of liver fluke EVs and suppress the proliferation and secretion of IL- 6 by cholangiocytes [33]. Other helminth proteins induce changes that reflect the hallmarks of cancer, including EMT phenotypes, proinflammatory cytokines, and repression of apoptosis [32, 35]. Type $2 \mathrm{~T}$ helper cell (Th2) responses induced by schistosome proteins including interleukin 4-inducing principle of schistosome eggs (IPSE) not only facilitate egress of the schistosome egg to the bladder lumen by modulating the granuloma but also appear to modulate vasculogenic and cellular responses conducive to neoplasia $[10,36]$. Targeting these or other parasite-host cell communicating proteins with vaccines may not only block helminth infections but deliver novel anticancer vaccines [11].

\section{Acknowledgments}

We thank Javier Sotillo, Michael Smout, Thewarach Laha, Victoria Mann, Nuno Value, José M. Costa and Banchob Sripa for fruitful discussions including assistance with illustrations. We acknowledge collaboration and support of colleagues in TOPIC, the Tomsk Opisthorchiasis Consortium, http://www.topic-global.org/

\section{References}

1. de Martel C, Ferlay J, Franceschi S, Vignat J, Bray F, Forman D, et al. Global burden of cancers attributable to infections in 2008: a review and synthetic analysis. Lancet Oncol. 2012; 13(6):607-15. https:// doi.org/10.1016/S1470-2045(12)70137-7 PMID: 22575588.

2. Humans IWGotEoCRt. Biological agents. Volume $100 \mathrm{~B}$. A review of human carcinogens. IARC Monogr Eval Carcinog Risks Hum. 2012; 100(Pt B):1-441. PMID: 23189750. 
3. Zhu L, Finkelstein D, Gao C, Shi L, Wang Y, Lopez-Terrada D, et al. Multi-organ Mapping of Cancer Risk. Cell. 2016; 166(5):1132-46 e7. https://doi.org/10.1016/j.cell.2016.07.045 PMID: 27565343.

4. Sripa B, Kaewkes S, Sithithaworn P, Mairiang E, Laha T, Smout M, et al. Liver fluke induces cholangiocarcinoma. PLoS Med. 2007; 4(7):e201. https://doi.org/10.1371/journal.pmed.0040201 PMID: 17622191.

5. Khuntikeo N, Loilome W, Thinkhamrop B, Chamadol N, Yongvanit P. A Comprehensive Public Health Conceptual Framework and Strategy to Effectively Combat Cholangiocarcinoma in Thailand. PLoS Negl Trop Dis. 2016; 10(1):e0004293. https://doi.org/10.1371/journal.pntd.0004293 PMID: 26797527.

6. Haswell-Elkins MR, Satarug S, Tsuda M, Mairiang E, Esumi H, Sithithaworn $P$, et al. Liver fluke infection and cholangiocarcinoma: model of endogenous nitric oxide and extragastric nitrosation in human carcinogenesis. Mutat Res. 1994; 305(2):241-52. PMID: 7510035.

7. Satarug $S$, Lang MA, Yongvanit $P$, Sithithaworn $P$, Mairiang $E$, Mairiang $P$, et al. Induction of cytochrome P450 $2 \mathrm{~A} 6$ expression in humans by the carcinogenic parasite infection, opisthorchiasis viverrini. Cancer Epidemiol Biomarkers Prev. 1996; 5(10):795-800. PMID: 8896890.

8. Brindley PJ, da Costa JM, Sripa B. Why does infection with some helminths cause cancer? Trends Cancer. 2015; 1(3):174-82. https://doi.org/10.1016/j.trecan.2015.08.011 PMID: 26618199.

9. Mitacek EJ, Brunnemann KD, Suttajit M, Martin N, Limsila T, Ohshima H, et al. Exposure to N-nitroso compounds in a population of high liver cancer regions in Thailand: volatile nitrosamine (VNA) levels in Thai food. Food Chem Toxicol. 1999; 37(4):297-305. PMID: 10418946.

10. Honeycutt J, Hammam $\mathrm{O}$, Fu CL, Hsieh MH. Controversies and challenges in research on urogenital schistosomiasis-associated bladder cancer. Trends Parasitol. 2014; 30(7):324-32. https://doi.org/10. 1016/j.pt.2014.05.004 PMID: 24913983.

11. Sripa B, Brindley PJ, Mulvenna J, Laha T, Smout MJ, Mairiang E, et al. The tumorigenic liver fluke Opisthorchis viverrini-multiple pathways to cancer. Trends Parasitol. 2012; 28(10):395-407. https:// doi.org/10.1016/j.pt.2012.07.006 PMID: 22947297.

12. Deenonpoe R, Mairiang E, Mairiang $P$, Pairojkul C, Chamgramol $Y$, Rinaldi G, et al. Elevated prevalence of Helicobacter species and virulence factors in opisthorchiasis and associated hepatobiliary disease. Sci Rep. 2017; 7:42744. https://doi.org/10.1038/srep42744 PMID: 28198451.

13. Jusakul $A$, Yongvanit $P$, Loilome $W$, Namwat $N$, Kuver R. Mechanisms of oxysterol-induced carcinogenesis. Lipids Health Dis. 2011; 10:44. https://doi.org/10.1186/1476-511X-10-44 PMID: 21388551.

14. Vale N, Gouveia MJ, Botelho M, Sripa B, Suttiprapa S, Rinaldi G, et al. Carcinogenic liver fluke Opisthorchis viverrini oxysterols detected by LC-MS/MS survey of soluble fraction parasite extract. Parasitol Int. 2013; 62(6):535-42. PMID: 23973383.

15. Knowles MA, Hurst CD. Molecular biology of bladder cancer: new insights into pathogenesis and clinical diversity. Nat Rev Cancer. 2015; 15(1):25-41. https://doi.org/10.1038/nrc3817 PMID: 25533674.

16. Shin K, Lim A, Odegaard JI, Honeycutt JD, Kawano S, Hsieh MH, et al. Cellular origin of bladder neoplasia and tissue dynamics of its progression to invasive carcinoma. Nat Cell Biol. 2014; 16(5):469-78. https://doi.org/10.1038/ncb2956 PMID: 24747439.

17. Van Batavia J, Yamany $T$, Molotkov A, Dan H, Mansukhani M, Batourina E, et al. Bladder cancers arise from distinct urothelial sub-populations. Nat Cell Biol. 2014; 16(10):982-91, 1-5. https://doi.org/10. 1038/ncb3038 PMID: 25218638.

18. Gouveia MJ, Santos J, Brindley PJ, Rinaldi G, Lopes C, Santos LL, et al. Estrogen-like metabolites and DNA-adducts in urogenital schistosomiasis-associated bladder cancer. Cancer Lett. 2015; 359(2):22632. https://doi.org/10.1016/j.canlet.2015.01.018 PMID: 25615421.

19. Yongvanit $P$, Pinlaor S, Bartsch H. Oxidative and nitrative DNA damage: key events in opisthorchiasisinduced carcinogenesis. Parasitol Int. 2012; 61(1):130-5. https://doi.org/10.1016/j.parint.2011.06.011 PMID: 21704729.

20. Fu CL, Odegaard JI, Herbert DR, Hsieh MH. A novel mouse model of Schistosoma haematobium egginduced immunopathology. PLoS Pathog. 2012; 8(3):e1002605. https://doi.org/10.1371/journal.ppat. 1002605 PMID: 22479181.

21. Honeycutt J, Hammam $\mathrm{O}$, Hsieh MH. Schistosoma haematobium egg-induced bladder urothelial abnormalities dependent on p53 are modulated by host sex. Exp Parasitol. 2015; 158:55-60. https://doi.org/ 10.1016/j.exppara.2015.07.002 PMID: 26160678.

22. Chala B, Choi MH, Moon KC, Kim HS, Kwak C, Hong ST. Development of Urinary Bladder Pre-Neoplasia by Schistosoma haematobium Eggs and Chemical Carcinogen in Mice. Korean J Parasitol. 2017; 55(1):21-9. https://doi.org/10.3347/kjp.2017.55.1.21 PMID: 28285503.

23. Thamavit W, Bhamarapravati N, Sahaphong S, Vajrasthira S, Angsubhakorn S. Effects of dimethylnitrosamine on induction of cholangiocarcinoma in Opisthorchis viverrini-infected Syrian golden hamsters. Cancer Res. 1978; 38(12):4634-9. PMID: 214229. 
24. Chan-On W, Nairismagi ML, Ong CK, Lim WK, Dima S, Pairojkul C, et al. Exome sequencing identifies distinct mutational patterns in liver fluke-related and non-infection-related bile duct cancers. Nat Genet. 2013; 45(12):1474-8. https://doi.org/10.1038/ng.2806 PMID: 24185513.

25. Jusakul A, Kongpetch S, Teh BT. Genetics of Opisthorchis viverrini-related cholangiocarcinoma. Curr Opin Gastroenterol. 2015; 31(3):258-63. https://doi.org/10.1097/MOG.0000000000000162 PMID: 25693006.

26. Andresen $\mathrm{K}$, Boberg KM, Vedeld HM, Honne H, Jebsen $\mathrm{P}$, Hektoen $\mathrm{M}$, et al. Four DNA methylation biomarkers in biliary brush samples accurately identify the presence of cholangiocarcinoma. Hepatology. 2015; 61(5):1651-9. https://doi.org/10.1002/hep.27707 PMID: 25644509.

27. Cancer Genome Atlas Research N. Comprehensive molecular characterization of urothelial bladder carcinoma. Nature. 2014; 507(7492):315-22. https://doi.org/10.1038/nature12965 PMID: 24476821.

28. Alexandrov LB, Ju YS, Haase K, Van Loo P, Martincorena I, Nik-Zainal S, et al. Mutational signatures associated with tobacco smoking in human cancer. Science. 2016; 354(6312):618-22. https://doi.org/ 10.1126/science.aag0299 PMID: 27811275.

29. van Tong H, Brindley PJ, Meyer CG, Velavan TP. Parasite Infection, Carcinogenesis and Human Malignancy. EBioMedicine. 2016. https://doi.org/10.1016/j.ebiom.2016.11.034 PMID: 27956028.

30. Smout MJ, Laha T, Mulvenna J, Sripa B, Suttiprapa S, Jones A, et al. A granulin-like growth factor secreted by the carcinogenic liver fluke, Opisthorchis viverrini, promotes proliferation of host cells. PLoS Pathog. 2009; 5(10):e1000611. https://doi.org/10.1371/journal.ppat.1000611 PMID: 19816559.

31. Li J, Razumilava N, Gores GJ, Walters S, Mizuochi T, Mourya R, et al. Biliary repair and carcinogenesis are mediated by IL-33-dependent cholangiocyte proliferation. J Clin Invest. 2014; 124(7):3241-51. https://doi.org/10.1172/JCl73742 PMID: 24892809.

32. Smout MJ, Sotillo J, Laha T, Papatpremsiri A, Rinaldi G, Pimenta RN, et al. Carcinogenic Parasite Secretes Growth Factor That Accelerates Wound Healing and Potentially Promotes Neoplasia. PLoS Pathog. 2015; 11(10):e1005209. https://doi.org/10.1371/journal.ppat.1005209 PMID: 26485648.

33. Chaiyadet S, Sotillo J, Smout M, Cantacessi C, Jones MK, Johnson MS, et al. Carcinogenic Liver Fluke Secretes Extracellular Vesicles That Promote Cholangiocytes to Adopt a Tumorigenic Phenotype. J Infect Dis. 2015; 212(10):1636-45. https://doi.org/10.1093/infdis/jiv291 PMID: 25985904.

34. Hemler ME. Tetraspanin functions and associated microdomains. Nat Rev Mol Cell Biol. 2005; 6 (10):801-11. https://doi.org/10.1038/nrm1736 PMID: 16314869.

35. Matchimakul P, Rinaldi G, Suttiprapa S, Mann VH, Popratiloff A, Laha T, et al. Apoptosis of cholangiocytes modulated by thioredoxin of carcinogenic liver fluke. Int J Biochem Cell Biol. 2015; 65:72-80. https://doi.org/10.1016/j.biocel.2015.05.014 PMID: 26007234.

36. Bernardo C, Cunha MC, Santos JH, da Costa JM, Brindley PJ, Lopes C, et al. Insight into the molecular basis of Schistosoma haematobium-induced bladder cancer through urine proteomics. Tumour Biol. 2016; 37(8):11279-87. https://doi.org/10.1007/s13277-016-4997-y PMID: 26951512. 